\title{
Das Projekt LohnSpiegel: Tatsächlich gezahlte Löhne und Gehälter
}

\author{
Reinhard Bispinck \\ Heiner Dribbusch \\ Fikret Öz
}

Seit 2004 führt das WSI-Tarifarchiv das Projekt „LohnSpiegel“ durch, das auf einer kontinuierlich laufenden Online-Erhebung von Daten zu den Arbeits- und Einkommensbedingungen von Arbeitnehmerinnen und Arbeitnehmern basiert. Die gewonnenen Daten werden den Besuchern der Website www.lohnspiegel.de in Form eines Lohn- und Gehaltschecks zur Verfügung gestellt. Sie bilden gleichzeitig einen Datensatz, der für wissenschaftliche Analysen genutzt wird. Welche Stärken, Schwächen und Potenziale hat dieser neue methodische Zugriff? Was haben wir jetzt schon aus den Erhebungen gelernt?

\section{Das Projekt "LohnSpiegel" und Wagelndicator}

Die LohnSpiegel-Datenbank ist entstanden aus dem europäischen Forschungsprojekt „Work and Life on the Web" (WOLIWEB), das von April 2004 bis März 2007 in acht europäischen Ländern durchgeführt und von der EU-Kommission gefördert wurde. Ziel von WOLIWEB war, Transparenz bei Löhnen und Gehältern zu fördern und Arbeitnehmerinnen und Arbeitnehmern, aber auch anderen Interessierten, die Möglichkeit zu geben, sich im Internet rasch über die aktuellen Arbeitsvergütungen zu informieren. Seitdem wird der LohnSpiegel vom WSI in eigener Verantwortung und in Kooperation mit der WageIndicator Foundation (Amsterdam) weitergeführt. ${ }^{1}$

Die Zahl der internationalen Partner des internationalen WageIndicator-Netzwerkes wurde im Anschluss an WOLIWEB kontinuierlich ausgebaut. Die technische Koordination wird von der WageIndicator Foundation fortgeführt und die akademische Leitung des Verbundes blieb beim Amsterdam Institute for Advanced Labour Studies (AIAS) an der Universität Amsterdam, das eng mit den angeschlossenen Forschungsinstituten und Gewerkschaften zusammenarbeitet. Zurzeit wird die Online-Befragung in 35 Ländern durchgeführt. Langfristig ist geplant, den WageIndicator auf 75 Staaten der Welt auszudehnen, um perspektivisch auch auf breiter Basis internationale Daten zu Löhnen und Gehältern zu bekommen.

Die Datenerhebung für den Lohnspiegel wie für die anderen WageIndicatorProjekte erfolgt als Online-Befragung. Beschäftigte in den beteiligten Ländern können als Besucher der entsprechenden Websites freiwillig und anonym länderspezifisch ausgestaltete Online-Fragebögen ausfüllen. Die zugrunde liegenden Fragebögen werden soweit möglich homogenisiert, erlauben aber auch besondere Anpassungen. Ausnahmsweise werden in einzelnen Ländern mit sehr geringer Verbreitung des Internets auch mittels Papierfragebögen Daten gesammelt. Die fortlaufende Erhebung von Daten ermöglicht eine regelmäßige Aktualisierung des Datenbestandes. Ausgefüllte Fragebögen werden in Amsterdam zentral gespeichert und bereinigt, neue Datenzusammenstellungen den beteiligten Ländern zur Auswertung in vierteljährlichen Abständen geliefert. Im Vergleich zu anderen Datensätzen in Deutschland (z. B. Sozio-oekonomisches Panel (SOEP), Betriebspanel des Instituts für Arbeitsmarktund Berufsforschung (IAB), IAB-Beschäftigtenstichprobe etc.) bietet der Lohnspiegel zusätzliche Auswertungsmöglichkeiten. Dies gilt insbesondere für vertiefende Analysen auf Berufebasis. Durch die kontinuierlich laufende Erhebung liefert der Lohnspiegel ständig aktuelle Daten.

Zentrale Untersuchungsfelder sind die Strukturen und die Differenzierung tatsächlich gezahlter Einkommen, die Arbeitszeitstrukturen und individuellen Arbeitszeit- präferenzen, betriebliche Umstrukturierungen sowie die Personalentwicklung und ihre Wahrnehmung seitens der Beschäftigten und schließlich die Auswirkungen der Tarifbindung auf Einkommens- und Arbeitsbedingungen und ihre Bewertung.

\section{Angebot, Nutzung, Transfer}

\subsection{INFOANGEBOTE: LOHN- UND GEHALTSCHECK}

Im Mittelpunkt des Internetangebots steht der Gehaltscheck mit Informationen zu

\footnotetext{
1 Unter der Internetadresse www.wageindicator.org finden sich nähere Informationen über das Projekt, den Forschungsverbund und über die Projektveröffentlichungen.
}

Reinhard Bispinck, Dr., Wissenschaftler im WSI und Leiter des WSI-Tarifarchivs in der Hans-Böckler-Stiftung.

e-mail: Reinhard-Bispinck@boeckler.de Heiner Dribbusch, Dr., Wissenschaftler im WSI in der Hans-Böckler-Stiftung. Arbeitsschwerpunkte: Industrielle Beziehungen, Arbeitskampfentwicklung. e-mail: Heiner-Dribbusch@boeckler.de Fikred Öz, Wissenschaftler im WSI in der Hans-Böckler-Stiftung. Arbeitsschwerpunkt: Projekt LohnSpiegel. e-mail: Fikret-Oez@boeckler.de 


\begin{tabular}{ll}
\hline Übersicht 1: Berufsgruppen im Gehaltscheck des LohnSpiegel \\
\hline Architekturberufe, Raumplanung & Bank- und Finanzberufe \\
Bauberufe & Büro und Verwaltung \\
Callcenter & Chemieberufe \\
Dienstleistungsberufe & Druckberufe \\
EDV/IT-Berufe & Elektroberufe \\
Gebäudereinigung & Gesundheitsberufe \\
Handel & Handwerksberufe \\
Hotel, Gaststätten, Tourismus & Ingenieurberufe \\
Journalistische Berufe & Lehrberufe \\
Marketing/Werbung & Medien/Gestaltung \\
Metallberufe & Nahrungsmittelverarbeitung \\
Rechtsberufe & Sicherheitsberufe \\
Sozialberufe & Spedition und Lager \\
Techniker & Transport/Verkehr \\
Weitere Wissenschaftsberufe & Wirtschaftswissenschaftler \\
Übersetzer, Dolmetscher & \\
\hline &
\end{tabular}

tatsächlich gezahlten Löhnen und Gehältern in rund 280 Berufen. Die Angaben stammen aus der laufenden Online-Umfrage, die ebenfalls auf dieser Internetseite angeboten wird. Kernanliegen des Lohnund Gehaltschecks ist, einen Beitrag zur Lohn- und Gehaltstransparenz zu leisten. Ziel ist es, den Nutzern der Website zu einer möglichst großen Anzahl von Berufen und Tätigkeiten gegliederte Informationen zu tatsächlich gezahlten Gehältern anzubieten. Zugleich ist der Lohn- und Gehaltscheck das zentrale Angebot, mit dem für die Website selbst und insbesondere für Teilnahme an der LohnSpiegel-Umfrage geworben wird. Die Umfrageteilnahme ist aber bewusst keine Voraussetzung, um den Lohn- und Gehaltscheck nutzen zu können.

In den Gehaltscheck werden all die Berufe aufgenommen, für die eine ausreichende Anzahl auswertbarer Online-Fragebögen vorliegt. In der Regel müssen hierfür mindestens 40 Fragebögen vorhanden sein. Die erhobenen Einkommensdaten werden mit Hilfe multivariater Analysen auf Grundlage ausgewählter Variablen für den Online-Gehaltscheck aufbereitet.

Der Lohn- und Gehaltscheck bietet zurzeit (Dezember 2009) Informationen zu rund 280 Berufen und Tätigkeiten aus 31 Berufsgruppen (Übersicht 1).

\subsection{DER LOHN- UND GEHALTSCHECK IM NETZ}

Aus unserer wissenschaftlichen Arbeit wissen wir, dass die tariflich vereinbarten Einkommen stark von den effektiv gezahlten Löhnen und Gehältern abweichen. Die Be- d.h. jeden Monat besuchen im Schnitt rund 80.000 Nutzer die Seite, jeden Tag sind es rund 2600. Die Webstatistik und die Anzahl der ausgefüllten Fragebögen ( $A b$ bildung 1) zeigen, dass sich der LohnSpiegel zu einem wichtigen Internetportal in Bezug auf Einkommensfragen in Deutschland entwickelt hat.

Die bereits in der Startphase des Projekts begonnene Medienkooperation wurde fortgeführt und ausgebaut. Zur Verbreitung des LohnSpiegels bestehen Kooperationsvereinbarungen mit verschiedenen Medienpartnern, die das Angebot von www.lohnspiegel.de in ihr eigenes OnlineAngebot integriert haben. Seit Beginn des Projekts LohnSpiegel im Jahr 2004 gibt es eine enge Kooperation mit dem Deutschen Gewerkschaftsbund (DGB) und allen Einzelgewerkschaften. Auf zahlreichen gewerkschaftlichen Internetseiten ist der LohnSpiegel verlinkt, zum Teil auch ausführlicher dargestellt. Daneben gibt es eine Zusammenarbeit mit der Website der Bundesagentur für Arbeit „Berufenet“ und dem Bundesfrauenministerium. Medienkooperationen existieren u.a. mit der Süddeutschen Zeitung, dem Stern, T-Online, Zeit.de, der Website Arbeitsrecht.de sowie verschiedenen Regionalzeitungen. Wichtiger Zweck ist es, über eine entsprechende Verlinkung beziehungsweise Einbindung der LohnSpiegel-Website die Internetpräsenz zu erhöhen und so vermehrte Zugriffe auf die Seite zu generieren. Im Gegenzug werden auf Anfrage den Kooperationspartnern Sonderauswertungen zur Verfügung gestellt.

Knapp die Hälfte der Besucher wird von anderen Internetseiten auf den LohnSpiegel gelenkt, ein knappes Drittel findet über Suchmaschinen zum LohnSpiegel, rund ein Fünftel der Besucher greift direkt auf die Website zu. Bei den verweisenden Websites spielen das WSI-Tarifariv sowie die gewerkschaftlichen Internetseiten eine zentrale Rolle: Rund 60 \% der Besucher, die von anderen Websites zum LohnSpiegel finden, kommen über diesen Zugang.

Bei den nicht gewerkschaftlichen Internetseiten steht das Internetangebot „Berufenet" der Bundesagentur für Arbeit mit weitem Abstand an der Spitze; knapp jeder Fünfte an den LohnSpiegel verwiesene Besucher kommt über diese Seite. Bei aktueller Berichterstattung über den LohnSpiegel spielt das Internetangebot der betreffenden Medien eine wichtige Rolle als Zugangsweg. 


\section{Datensatz und Methoden}

\subsection{STRUKTUR DES DATENSATZES}

Die Datenerhebung für den LohnSpiegel erfolgt mittels einer kontinuierlich laufenden selbst administrierten Online-Befragung. Die Entwicklung des jährlichen Datenbestandes zeigt eine Stabilisierung des Zugangs, die jährlich zwischen 25.000 und 30.000 Fragebögen für Analysen zur Verfügung erwarten lässt (Abbildung 1).

Insgesamt stehen bisher (September 2009) ca. 150.000 auswertbare Datensätze zur Verfügung (Tabelle 1), die als Basis für Datenanalysen dienen. Auswertungen können sich auf den gesamten Datensatz oder beliebig definierte zeitliche Teilsegmente des Datensatzes beziehen.

Arbeitnehmer bilden als wichtigste Zielgruppe des LohnSpiegels mit einem Anteil von rund $91 \%$ die größte Personengruppe in der Datenbank (vgl. zur Struktur Tabelle 2). Entsprechend konzentrieren sich auf sie auch die bisherigen Analysen. Berufsgruppenspezifische Auswertungen stehen bisher im Mittelpunkt. Die LohnSpiegel-Datenbank liefert Angaben über etwa 600 Berufe.

\subsection{METHODISCHE PROBLEME UND ANSÄTZE}

Verglichen mit anderen Methoden steckt die Online-Befragung bezüglich ihrer Verbreitung noch in ihrem Anfangsstadium und hat wie alle Befragungsmethoden spezifische Vorteile, aber auch Beschränkungen (vgl. zur Diskussion Andralojc et al. 2007; Tijdens 2004). Wie Sue/Ritter (2007) anmerken, berührt die Methodendiskussion dabei vielfach Punkte, die auch bei früheren Befragungsinnovationen, so bei der Einführung von Post- und Telefonbefragungen, eine Rolle spielten.

Online-Befragungen haben in den letzten Jahren in dem Maße an Bedeutung gewonnen, in dem sich die private Nutzung von Computern verbreitet und ihr alltäglicher Gebrauch Akzeptanz gefunden hat. Die Voraussetzung jeder Teilnahme an Online-Befragungen ist der Zugang zum Internet. Dieser ist in den letzten Jahren in Deutschland kontinuierlich gestiegen. Nutzten 1997 noch lediglich 6,5 \% der Bevölkerung das Internet, so waren es bei

Abb. 1: Anzahl* der Fragebögen im LohnSpiegel 4/2004 bis 1/2009

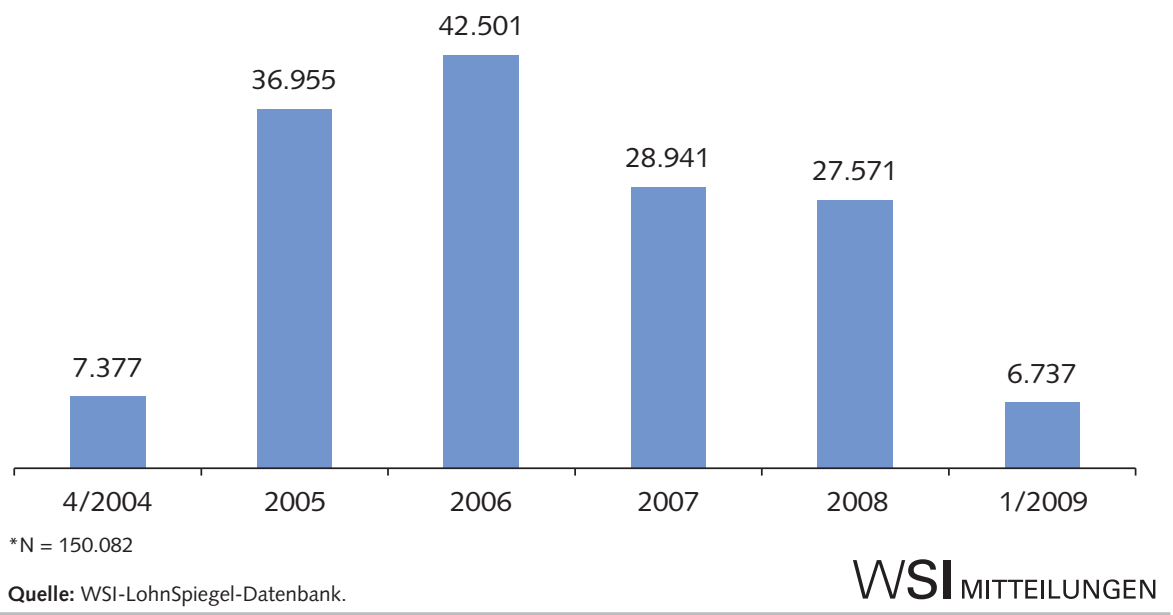

\section{Tabelle 1: Teilnehmerinnen und Teilnehmer im LohnSpiegel} 4/2004-1/2009

\begin{tabular}{lr}
\hline Arbeitnehmerinnen und Arbeitnehmer & 136.547 \\
Mithelfende Familienangehörige - Tätigkeit im eigenen Familienbetrieb & 369 \\
Selbstständige - Freelancer & 4.196 \\
Arbeitslose - Arbeitssuchende & 1.786 \\
Krankengeldbezieher - Erwerbsunfähiger & 133 \\
Schüler - Studenten & 1.886 \\
Auszubildende - Trainees & 2.457 \\
Teilnehmer an einer Beschäftigungsmaßnahme & 102 \\
Bisher nicht berufstätig gewesen & 793 \\
Sonstiges & 1.813 \\
\hline Insgesamt & 150.082 \\
\hline &
\end{tabular}

Beginn des LohnSpiegelprojektes bereits $55,3 \%$ und 2009 mit 67,1\% bereits gut zwei Drittel der Bevölkerung (ARD/ZDFOnline-Studie 2009). Das Nutzungsverhalten unterscheidet sich nach Geschlechtern. So nutzen 76,1 \% der Männer das Internet. Bei den Frauen sind es nur 62,4 \%. ((N)Onliner Atlas 2009).

Unter der Zielgruppe des LohnSpiegels, den Berufstätigen, liegt der Anteil der zumindest gelegentlichen Nutzer nach Angaben der Atlas (N)Onliner Studie 2009 mit $83 \%$ noch einmal höher. In den Altersgruppen von 14- bis 49-Jährigen steigt er sogar auf $85 \%$. Die Berufstätigen ab 50 Jahren liegen mit immerhin 70,4\% über dem Bundesdurchschnitt und weit über dem Anteil von Nicht-Berufstätigen $(32,8 \%)$ in der gleichen Altersgruppe.

Durch die weitere Verbreitung des Internetzugangs lässt sich erwarten, dass größere Teile der Bevölkerung für OnlineBefragungen erreichbar werden. Generelle Probleme der Online-Befragungen sind jedoch nicht nur auf den Internetzugang beschränkt. Im Vordergrund der Diskussion stehen vor allem methodische Aspekte.

Der Anteil der Online-Befragungen in Deutschland ist zwischen 2000 and 2008 von $3 \%$ auf $31 \%$ gestiegen (ADM 2009). In den USA wird mittlerweile jede dritte Befragung online durchgeführt. Seit 2004 haben Online-Interviews schriftliche Befragungen verdrängt und als Befragungsform nach den telefonischen Befragungen den zweiten Platz eingenommen (Maurer/ Jandura 2009). Es wird erwartet, dass für die Mehrheit aller Befragungen in Zukunft die Möglichkeiten des World Wide Web genutzt werden (Jackob et al. 2009).

$\mathrm{Zu}$ den unmittelbaren Vorteilen internetbasierter Online-Umfragen zählen Geschwindigkeit, Kostenersparnis und potenzielle Internationalität (Fischer 2005; Andralojc et al. 2007). Sie erlauben orts- und zeitunabhängige Befragungen sowie eine kontinuierliche, standardisierbare Datenerfassung. Multimediale Hilfsmittel können problemlos in die Befragungen integriert werden. Interviewereffekte und Auf- 
Tabelle 2: Das Gesamtsample in der LohnSpiegel-Datenbank 4/2004 und 1/2009 - Arbeitnehmerinnen und Arbeitnehmer -

\begin{tabular}{|c|c|c|}
\hline & Anzahl & in \% \\
\hline \multicolumn{3}{|l|}{ Geschlecht } \\
\hline Männer & 86.610 & 64,1 \\
\hline Frauen & 48.409 & 35,9 \\
\hline Gesamt & 135.019 & 100,0 \\
\hline \multicolumn{3}{|l|}{ OST/WEST } \\
\hline Ost inkl. Berlin & 18.916 & 14,6 \\
\hline West & 110.647 & 85,4 \\
\hline Gesamt & 129.563 & 100,0 \\
\hline \multicolumn{3}{|l|}{ Wirtschaftssektoren } \\
\hline Produzierende Gewerbe & 40.689 & 30,1 \\
\hline Energie- und Wasserversorgung, Entsorgung, Recycling & 4.041 & 3,0 \\
\hline Baugewerbe & 9.555 & 7,1 \\
\hline $\begin{array}{l}\text { Handel, Instandhaltung und Reparatur von Kraftfahrzeugen } \\
\text { und Gebrauchsgütern }\end{array}$ & 13.921 & 10,3 \\
\hline Hotel- und Gaststättengewerbe, Gastronomie & 3.196 & 2,4 \\
\hline Transport, Logistik, Post u. Telekommunikation & 7.606 & 5,6 \\
\hline Kredit- und Versicherungsgewerbe & 7.196 & 5,3 \\
\hline Öffentlicher Dienst, Verbände & 7.130 & 5,3 \\
\hline Erziehung und Unterricht, Forschung und Entwicklung & 4.229 & 3,1 \\
\hline Gesundheits- und Sozialwesen, Pflegedienstleistungen & 11.506 & 8,5 \\
\hline Sonstige wirtschaftliche Dienstleistungen & 26.006 & 19,3 \\
\hline Gesamt & 135.075 & 100,0 \\
\hline \multicolumn{3}{|l|}{ Betriebsgröße } \\
\hline Unter 100 & 64.589 & 47,8 \\
\hline 100 bis 500 & 30.802 & 22,8 \\
\hline Über 500 & 39.629 & 29,4 \\
\hline Gesamt & 135.020 & 100,0 \\
\hline \multicolumn{3}{|l|}{ Tarifbindung im Betrieb } \\
\hline Kein Tarifvertrag & 54.176 & 45,4 \\
\hline Mit Tarifvertrag & 65.071 & 54,6 \\
\hline Gesamt & 119.247 & 100,0 \\
\hline \multicolumn{3}{|l|}{ Arbeitszeit } \\
\hline Teilzeit & 12.618 & 9,3 \\
\hline Vollzeit & 122.344 & 90,7 \\
\hline Gesamt & 134.962 & 100,0 \\
\hline \multicolumn{3}{|l|}{ Arbeitsverhältnis } \\
\hline Befristed & 15.735 & 11,7 \\
\hline Unbefristed & 119.300 & 88,3 \\
\hline Gesamt & 135.035 & 100,0 \\
\hline \multicolumn{3}{|l|}{ Höchster Schulabschluss } \\
\hline Schule ohne Abschluss verlassen & 708 & 0,5 \\
\hline Volksschul-/Hauptschulabschluss/8. Klasse & 15.550 & 11,5 \\
\hline Realschule/Mittlere Reife/10. Klasse & 37.667 & 27,9 \\
\hline Fach(hoch)schulreife/Fachoberschule & 17.130 & 12,7 \\
\hline Abitur/Hochschulreife & 17.761 & 13,2 \\
\hline Uni/TH/FH & 41.845 & 31,0 \\
\hline Promotion & 4.224 & 3,1 \\
\hline Gesamt & 134.885 & 100,0 \\
\hline
\end{tabular}

wand der Dateneingabe entfallen. Ein weiteres Plus liegt darüber hinaus in der hohen Akzeptanz bei den Befragten, die aus der vollständigen Freiwilligkeit (jederzeit ist der Abbruch der Befragung möglich) sowie aus hoher Anonymität und selbstbestimmter Wahl des Zeitpunkts resultiert. Durch entsprechendes Posting sowie über breite Medienarbeit können sehr breite Zielgruppen erreicht werden.
Diesen Vorteilen stehen, darüber herrscht in der Forschung Einigkeit, einige spezifische Einschränkungen gegenüber. Deren wichtigste ist sicherlich das $\mathrm{Zu}-$ gangssystem. Diese Stichprobenproblematik schließt ein, dass die Identität der Befragten unklar bleibt und Mehrfachteilnahmen nicht vollständig auszuschließen sind. Damit verknüpft stellt sich die Frage der Repräsentativität.
Diese wird generell als einer der größten Nachteile der Online-Befragungen angesehen. Von Repräsentativität ist die Rede, wenn anhand einer Stichprobe zutreffende und generalisierbare Aussagen über die Grundgesamtheit getroffen werden können. Bei fehlender Repräsentativität ist keine Verallgemeinerung der Befragungsergebnisse auf die Grundgesamtheit möglich. Bei zufallsbasierten Verfahren ist die Auswahlwahrscheinlichkeit bekannt und für alle Einheiten im Auswahlrahmen (Sampling Framework) größer als Null. Die Voraussetzungen für die induktive Statistik sind aber in den Online-Befragungen meist nicht erfüllt (Kutsch 2007). Diese Unzulänglichkeiten bilden die Ursachen der Auswahlverzerrungen (Abbildung 2):

(1) (Non-)Coverage: Nicht alle Personen sind übers Internet erreichbar. Die Übereinstimmung mit der Zielpopulation (z. B. Arbeitsmarkt, Erwerbstätige in Deutschland etc.) ist unsicher, d.h. die Struktur der Teilnehmer unterscheidet sich möglicherweise systematisch von der Struktur der Zielpopulation. Personen mit und ohne Internetzugang unterscheiden sich z. B. nach Gehalt, Alter und Bildung.

(2) Self-Selection: Die Teilnehmer entschließen sich, im Gegensatz zur Vorgehensweise bei traditionellen Befragungen, selbst zur Teilnahme und werden nicht zufällig aus einer Grundgesamtheit ausgewählt. Online-Befragungen sind also ein nichtzufallsbasiertes Verfahren (non-probability sampling). Es existiert kein statistisches Modell, um Messfehler theoretisch berechnen zu können. Wenn sich die Teilnehmer und Nichtteilnehmer in Bezug auf zu untersuchenden Merkmale stark unterscheiden, könnte dies zur starken Verzerrungen und zu größeren Messfehlern führen.

(3) Non-Response: Nicht alle Personen beenden den Fragebogen. Problematisch ist dies vor allem, wenn die Ausfälle mit den Merkmalen korrelieren, die untersucht werden.

Zahlreiche Studien zeigen, dass die Personen, die an Online-Befragungen teilnehmen, tendenziell folgende Merkmale aufweisen: intensive Nutzung des Internets und anderer technischer Geräte, höhere Bildung, höhere politische und soziale Aktivität (Kaczmirek 2009). 


\subsection{REPRÄSENTATIVITÄTSLÜCKEN}

Die Stichprobenproblematik zeigt sich im Vergleich des LohnSpiegel-Datensatzes mit der Statistik zur sozialversicherungspflichtigen Beschäftigung, die von der Bundesagentur für Arbeit erhoben wird (Tabelle 3).

Hierbei offenbaren sich teils deutliche Repräsentativitätslücken. So sind Frauen im LohnSpiegeldatensatz unterrepräsentiert, ebenso Beschäftigte aus Ostdeutschland. Beschäftigte aus Großbetrieben sind überproportional vertreten. Besonders ausgeprägt sind die personengruppenbezogenen Unterschiede beim Merkmal Bildungsabschluss, wo eine Unterrepräsentanz von Beschäftigten mit Volks-, Haupt-, und Mittelschulabschluss einer starken Überrepräsentanz der Hochschulabsolventen gegenübersteht. Letzteres ist offenbar durch die gewählte Methode der Datensammlung begünstigt. Wie unsere $\mathrm{Zu}-$ griffsstatistiken zeigen, wird die LohnSpiegel-Website insbesondere tagsüber und an Werktagen aufgesucht. Dabei beteiligen sich überdurchschnittlich häufig solche Beschäftigtengruppen an der Umfrage, die entweder beruflich mit dem Internet $\mathrm{zu}$ tun haben oder aber an ihrem Arbeitsplatz Zugang zum World Wide Web haben. In diesen Beschäftigtengruppen finden sich ganz offensichtlich besonders viele Akademiker.

Ein großes Gewicht hat die Altersgruppe von 25 bis unter 45 Jahren im LohnSpiegeldatensatz, unterrepräsentiert sind dagegen die übrigen Altersgruppen (Abbildung 3). Dieser altersbezogene Bias ist im Projektverlauf geringer geworden. So sank der Anteil der Altersgruppe (25 bis unter 45) von $73,8 \%$ im Jahre 2005 auf $62,9 \%$ im Jahre 2009. Die Erreichbarkeit der Altersgruppe über 50 Jahre stellt auch in anderen Internetbefragungen ein Problem dar. Die Gruppe unter 25 Jahre weist hingegen im Vergleich zu anderen Altersgruppen eine höhere Internetnutzungsintensität auf, die sich jedoch nicht in der Nutzung des LohnSpiegels niederschlägt. $\mathrm{Zu}$ vermuten ist u.a., dass diese Altersgruppe über die Websites der führenden MedienKooperationspartner nur unzureichend erreicht wird.

Bei der Analyse des Datensatzes und insbesondere bei der Interpretation der Ergebnisse müssen die Repräsentativitätslücken und Probleme der Erhebungsmethodik berücksichtigt werden. Sie stellen

\section{Abb. 2: Stichprobenproblem in Online-Befragungen}

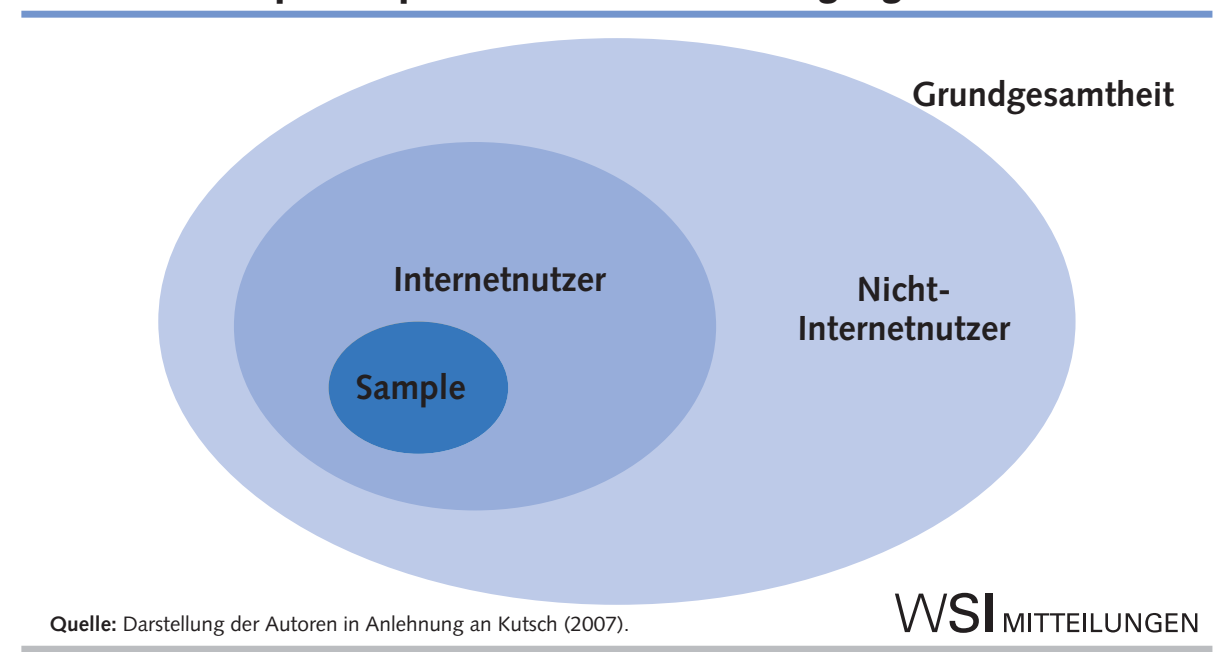

sich stärker bei Querschnittsanalysen als bei berufs- oder berufsgruppenbezogenen Auswertungen. Mittlerweile existieren zahlreiche Ansätze, um die Repräsentativitätsprobleme der Online-Befragungen zu vermindern. Um Aussagen über die Genauigkeit zu treffen, werden andere Mittel, z. B. über den Vergleich von zentralen Schätzern mit anderen Studien, in die Analyse einbezogen.

Diese Problematik der Online-Befragungen ist sowohl dem WageIndicator- wie auch dem LohnSpiegel-Projekt von Beginn an bewusst gewesen. Kontinuierlich wird daher der vorhandene Datensatz auf Vergleichbarkeit und Repräsentativität hin analysiert. Eine Anwendung des Gewichtungsverfahrens „Propensity Score Adjustment" anhand der SOEP-Daten lieferten für den LohnSpiegel keine zufriedenstellenden Ergebnisse. Ungewichtete SOEPDaten selbst weisen größere zielgruppenspezifische Repräsentationslücken auf. Im
Mittelpunkt eines vom WSI organisierten eintägigen wissenschaftlichen Workshops „Webbasierte Befragungen: Erfahrungen, Probleme, Perspektiven am Beispiel von WageIndicator und LohnSpiegel" im Juni 2009 wurden die genannten methodischen Probleme der Online-Befragungen diskutiert. Im Vordergrund unserer methodischen Bemühungen werden künftig folgende Punkte stehen:

- Durch eine zielgruppenspezifische Marketingstrategie sollen bisher wenig erreichte und schwer erreichbare Zielgruppen zu einer Teilnahme motiviert werden.

- Das Verfahren des Propensity Score Adjustment soll mit Referenzstudien überprüft und erprobt werden.

- Im Rahmen des EU-Netzwerkes „COST“ werden u. a. Gewichtungsproblematik und Lösungsansätze in Bezug auf WageIndicator und LohnSpiegel diskutiert und evaluiert.

\begin{tabular}{lcc}
\hline Tabelle 3: LohnSpiegel und Beschäftigtenstatistik im Vergleich - in \% - \\
\hline $\begin{array}{l}\text { LohnSpiegel } \\
\text { Arbeitnehmer }\end{array}$ & $\begin{array}{c}\text { Bundesagentur für Arbeit* } \\
\text { Beschäftigtenstatistik }\end{array}$ \\
\hline Frauen & 35,1 & 45,0 \\
Männer & 64,9 & 55,0 \\
Ost & 13,4 & 19,1 \\
West & 86,6 & 80,9 \\
Betriebsgröße & & \\
unter 100 AN & 46,5 & 52,9 \\
100-500 AN & 23,0 & 26,1 \\
über 500 AN & 30,5 & 21,0 \\
Bildungsabschluss & & 67,9 \\
Volks-/Hauptschulabschl./Mittl. Reife & 37,1 & 9,8 \\
Hochschulabschluss (FH, TH, Uni) & 31,7 & WSI MITTEILUNGEN \\
\hline * Stand: September 2007, Sozialversicherungspflichtig Beschäftigte. & & \\
Quelle: WSI-LohnSpiegel-Datenbank; Bundesagentur für Arbeit. & &
\end{tabular}


Abb. 3: Altersgrößenklassen: Lohnspiegel und BA-Statistik - in $\%-$

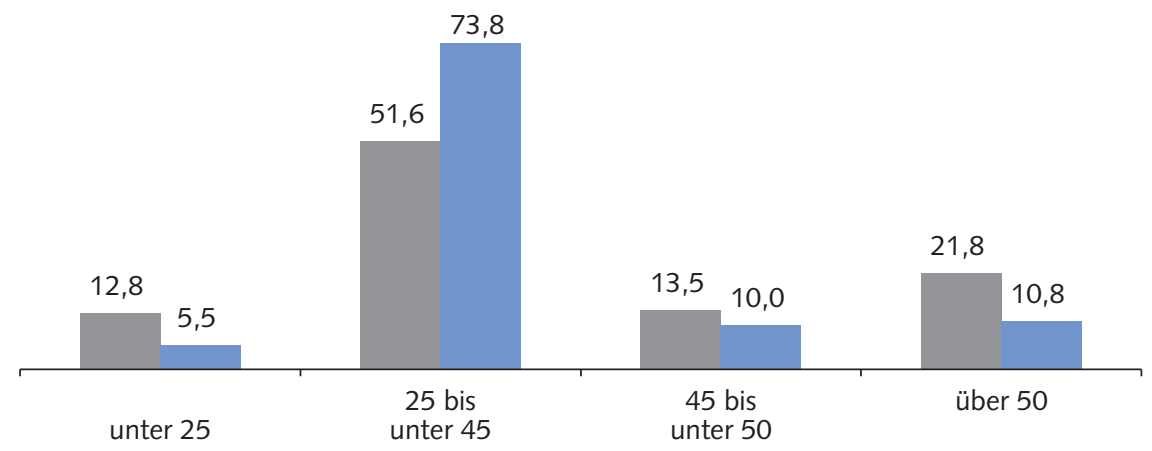

BA-Statistik $\square$ LohnSpiegel

WSI MITTELUNGEN

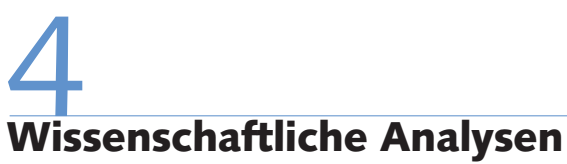

Wesentliches Ziel des LohnSpiegels wie auch des gesamten WageIndicator-Verbundes ist die wissenschaftliche Auswertung der nationalen wie internationalen $\mathrm{Da}$ tensätze in den zentralen Untersuchungsfeldern Einkommen, Arbeitszeit und Arbeitsbedingungen. Dabei können drei Untersuchungsebenen unterschieden werden: Detaillierte Berufsanalysen, Berufsübergreifende Analysen von Querschnittsthemen, internationale Vergleichsstudien in den genannten Untersuchungsfeldern.

\subsection{BERUFSBEZOGENE ANALYSEN}

Eine der Stärken des LohnSpiegel-Datensatzes liegt in den berufsbezogenen Analysen. Für umfangreichere Analysen bieten sich sowohl einzelne Berufe mit hohen Besetzungszahlen wie auch ganze Berufsgruppen an. Die bisherige Auswahl der Berufe war außerdem durch ein besonderes Interesse an der Einkommenssituation in klassischen wie neueren Angestelltentätigkeiten geleitet. Detaillierte Untersuchun- gen galten deshalb folgenden Berufsgruppen: Bürokaufleute (Öz/Bispinck 2008a), Bankkaufleute (Öz/Bispinck 2009a), Techniker (Öz/Bispinck 2009b), Ingenieure (Öz/Bispinck 2008b) sowie IT-Systemadministratoren (Gückelhorn et al. 2009).

Für alle Berufsanalysen gilt, dass zunächst eine Vergleichbarkeit der Einkommen hergestellt werden muss: Aus den vorliegenden Verdienst- und Arbeitszeitangaben wird deshalb zunächst ein rechnerischer Stundenlohn ermittelt, der dann auf Basis standardisierter Wochenarbeitszeiten auf Monatsverdienste hochgerechnet wird. Sonderzahlungen werden dabei nicht berücksichtigt. Auch die Auswertungskriterien werden soweit wie möglich standardisiert. Sie umfassen: Branchenzugehörigkeit, Region (West-Ost), Betriebsgröße, Tarifbindung, Berufserfahrung, Arbeitszeit (Vollzeit-Teilzeit), Geschlecht. Hinzu kommen verschiedene Aspekte der Arbeitsbedingungen wie z. B. Arbeitsbelastung, Arbeitsplatzsicherheit, Weiterbildung.

Ein Vergleich der bisherigen Berufsanalysen zeigt unabhängig von der unterschiedlichen absoluten Höhe der jeweiligen Monatseinkommen eine relativ ähnliche Binnendifferenzierung der Einkommen (Tabelle 4): Das fünfte Dezil beträgt

\begin{tabular}{lcccccc}
\multicolumn{7}{l}{ Tabelle 4: Einkommendezile in Euro und ihre Relation - in \% - } \\
\hline Beruf & 1. Dezil & 5. Dezil & 9. Dezil & $\mathbf{5 / 1}$ & $\mathbf{9 / 5}$ & $\mathbf{9 / 1}$ \\
\hline Bankkaufleute & 2.045 & 3.323 & 5.362 & 1,62 & 1,61 & 2,62 \\
Bürokaufleute & 1.235 & 1.942 & 3.178 & 1,57 & 1,63 & 2,57 \\
Ingenieure & 2.609 & 4.081 & 6.100 & 1,56 & 1,50 & 2,34 \\
IT-Systemadministrator & 1.922 & 3.040 & 4.485 & 1,53 & 1,48 & 2,25 \\
Techniker & 1.900 & 3048 & 4.549 & 1,60 & 1,49 & 2,39 \\
\hline
\end{tabular}

Quelle: WSI-LohnSpiegel-Datenbank.

WSI MITTEILUNGEN etwa das 1,5- bis 1,6-Fache des 1. Dezils, die Relation des 9. zum 5. Dezils liegt ebenfalls in etwa in diesem Bereich.

\subsection{UNTERSUCHUNGEN VON QUERSCHNITTSTHEMEN}

Aufgrund des vergleichsweise breiten Spektrums des Online-Fragebogens bieten sich ganz unterschiedliche Themen zur Untersuchung an, die u.a. von den Auswirkungen von Restrukturierungsmaßnahmen über Fragen der Weiterbildung bis hin zu berufsund branchenübergreifenden Arbeitszeitfragen reichen. Ein regelmäßig und besonders genau untersuchtes Themenfeld sind bisher die geschlechtsspezifischen Lohnund Einkommensunterschiede.

In einer Reihe einzelner Auswertungen (z. B. Bispinck et al. 2007) wie auch bei einer detaillierten Analyse für das Bundesfrauenministerium zur Situation von Berufsanfängerinnen (Bispinck et al. 2009) sind wir den Einkommensunterschieden zwischen Frauen und Männern nachgegangen. Dabei bestätigte sich der auch aus anderen Untersuchungen bekannte geschlechtsspezifische Einkommensabstand (Gender Pay Gap), auch wenn dieser in einzelnen Berufen unterschiedlich ausgeprägt ist. Differenziert man die Beschäftigten nach dem in einzelnen Berufen vorhandenen Frauenanteil, so bestätigt sich erneut der Befund, dass in Berufen mit besonders hohem Frauenanteil im Durchschnitt ein geringeres Einkommen gezahlt wird. Dieses geringere Einkommensniveau geht jedoch nicht zwangsläufig mit einem höheren Gender Pay Gap einher. Dieser liegt in den Berufen unseres Samples, die einen Frauenanteil von bis zu $60 \%$ aufweisen, etwas über $18 \%$, verringert sich aber in der Gruppe der Berufe mit einem Frauenanteil von über $60 \%$ auf knapp $14 \%$.

In der Studie wurde auch der Einfluss personenbezogener (Geschlecht, Alter, Ausbildung, Berufserfahrung, Kinder), betriebsbezogener (Wirtschaftszweig, Betriebsgröße, Frauenanteil, Tarifbindung), funktionaler (Vorgesetztenposition, Voll/Teilzeit) sowie regionaler Faktoren (Ost/ West) auf den Gender Pay Gap untersucht. Im Gesamtsample erklären diese Faktoren rund $60 \%$ der Lohndifferenz zwischen Männern und Frauen, d.h. 40 \% bleiben unerklärt. Bei den Berufsanfängern erklären diese Faktoren knapp 57 \% der Differenz. Den stärksten Beitrag liefern die personenbezogenen, gefolgt von den be- 
triebsbezogenen Faktoren. Sie erklären im Gesamtsample rund $44 \%$ und bei den Berufsanfängern sogar $54 \%$ der Lohndifferenz.

Insgesamt bestätigen unsere Studien, dass Frauen bereits in den ersten Berufsjahren finanziell gegenüber Männern benachteiligt werden. Dieser geschlechtsspezifische Einkommensrückstand lässt sich vollständig weder auf unterschiedliche Bildungsvoraussetzungen noch auf eine spezifische Berufswahl zurückführen. Die Ergebnisse verweisen vielmehr auf das Fortbestehen geschlechtspezifischer Lohndiskriminierung.

\subsection{INTERNATIONALE VERGLEICHSSTUDIEN}

Das WageIndicator-Projekt bietet sich wegen der einheitlichen Grundkonzeption der Fragebögen für komparative Untersuchungen an. Eine bereits Ende 2006 durchgeführte Untersuchung widmete sich dem für das WSI besonders interessanten Untersuchungsthema der Tarifbindung in acht europäischen Ländern (Dribbusch et al. 2007). Dabei bestätigt sich zunächst das bekannte Bild einer großen Spreizung der Tarifbindung von $14 \%$ in Polen über $29 \%$ in Großbritannien bis zu $88 \%$ in Finnland. In Deutschland korreliert die Ta- rifbindung stark mit der Betriebsgröße, ein Zusammenhang der auch durch multivariate Analysen bestätigt wird. Allerdings ist dieser Zusammenhang in anderen Ländern deutlich weniger ausgeprägt. Besonders ausgeglichen ist die Tarifbindung in Dänemark und Finnland. In allen Ländern fanden wir einen positiven Zusammenhang zwischen der Tarifbindung und der Gewerkschaftsmitgliedschaft der Beschäftigten - besonders ausgeprägt in den Ländern mit vergleichsweise geringer Tarifbindung.

Andere international ausgerichtete Vergleichsstudien innerhalb des WageIndicator-Netzwerkes beschäftigten sich u. a. mit Restrukturierungen innerhalb multinationaler Konzerne (Klaveren/Tijdens 2009), verschiedenen Aspekten der Teilzeitarbeit (Michon 2007), guter Arbeit (Öz 2008) sowie Belastungen am Arbeitsplatz (Klaveren/Tijdens 2007). Für den Internationalen Gewerkschaftsbund wurde ein breit angelegter Vergleich zum Gender Pay Gap ausgearbeitet (Chubb et al. 2008).

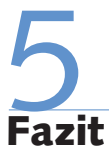

In Deutschland hat sich der LohnSpiegel mittlerweile als das führende nicht-kom- merzielle Angebot im Bereich der Lohnund Gehaltsauskunft und als anerkanntes Informationsportal zu Einkommensfragen etabliert.

Die im Rahmen des Projekts genutzte Methode der Online-Befragung hat sich seit einigen Jahren zu einem weit verbreiteten Instrument der sozialwissenschaftlichen Forschung entwickelt. Mit rund 150.000 Datensätzen liegt inzwischen ein umfangreicher Datensatz vor, der für vielfältige Analyse- und Forschungszwecke genutzt werden kann und wird. Die Einbindung in das WageIndicator-Netzwerk bietet überdies die Möglichkeit zu international vergleichenden Analysen. Die ständige Fortführung sichert dabei eine kontinuierliche Aktualisierung des Datenbestandes.

Dass die so gewonnenen Daten nicht nur für einen nutzerorientierten Lohnund Gehaltscheck, sondern auch für wissenschaftliche Analysen ertragreich sind, steht nach den bisherigen Erfahrungen außer Zweifel. Allerdings bleibt eine Reihe von methodischen Problemen, die insbesondere mit den Repräsentativitätslücken der Stichprobe und auch mit der Selbstselektion zusammenhängen. Die methodische Forschung auf diesem Gebiet steckt noch im Anfangsstadium.

\section{LITERATUR}

Andralojc, M./Osse, P./Dragstra, D./Kevätsalo, K./Oy, K./Tijdens, K. (2007): Evaluation report on Internet surveying and web visitors monitoring, Amsterdam; online: http://www.wageindicator.org/documents/ publicationslist/WOLIWEBinternet07 Arbeitskreis Deutscher Markt- und Sozialforschungsinstitute e. V. (ADM) (2009): Zahlen; online: www.adm-ev.de. (abgerufen am: 20.07.2009)

ARD/ZDF (2009): ARD/ZDF-Online Studie URL: www.ard-zdfonlinestudie.de. (abgerufen am 25.08.2009)

Bethlehem, J. (2009): Applied Survey Methods: A Statistical Perspective, Wiley Series in Survey Methodology, New Jersey

Bispinck, R./Dribbusch, H./Öz, F. (Mitarbeit) (2007): Germany Collective bargaining coverage and gender pay gap, Amsterdam; online: http://www.wageindicator.org/documents/publicationslist/ WOLIWEBinternet07
Bispinck, R./Dribbusch, H./Öz, F. (2009): Geschlechtsspezifische Lohndifferenzen nach dem Berufsstart und in der ersten Berufsphase - Eine Analyse von Einkommensdaten auf Basis der WSI-LohnSpiegel-Datenbank in Deutschland und im europäischen Vergleich - Projektbericht für das Bundesministerium für Familie, Senioren, Frauen und Jugend (BMFSFJ), Berlin

Chubb, C./Melis, S./Potter, L./Storry, R. (2008): Gender Pay Gap Report for the International Trade Union Confederation, IDS, London Dribbusch, H./Bispinck, R./Klaveren, M. v./Tijdens, K. (2007): Exploring collective bargaining coverage in eight EU member states, Amsterdam; online: http://www.wageindicator.org/documents/publicationslist/ WOLIWEBCollBar07

Fischer, M. (2005): Möglichkeiten sozialwissenschaftlicher Surveys im Internet. Stand und Folgerungen für Online-Befragungen, Universität Konstanz (Hrsg.): Hefte zur Bildungs- und Hochschulforschung 46 
Gückelhorn, C./Bispinck, R./Dribbusch, H./Öz, F. (2009): Was verdienen IT-Systemadministrator/innen? Eine Analyse von Einkommensdaten auf Basis der WSI-LohnSpiegel-Datenbank, Arbeitspapier 03, Düsseldorf Jackob, N./Schoen, H./Zerback, T. (Hrsg.): (2009): Sozialforschung im Internet: Methodologie und Praxis der Online-Befragung, Wiesbaden Kaczmirek, L. (2009): Rekrutierungsstrategien in Online-Befragungen und Ihre Auswirkungen auf die Datenqualität unter besonderer Berücksichtigung des Wageindicator. Präsentation im Workshop am 24.06. im WSI: „Webbasierte Befragungen: Erfahrungen, Probleme, Perspektiven am Beispiel von Wagelndicator und LohnSpiegel “

Klaveren, M. van/Tijdens K. G. (2007): Work-related stress, WIBAR report 6, Amsterdam; online: http://www.wageindicator.org/documents/ publicationslist/WIBARWorkStress

Klaveren, M. van/Tijdens, K. (2009): Reorganisations in multinational enterprises. Amsterdam; online: http://www.wageindicator.org/documents/publicationslist/wp25datameasurment

Kutsch, H. B. (2007): Repräsentativität in der Online-Marktforschung: Lösungsansätze zur Reduktion von Verzerrungen bei Befragungen im Internet, Electronic Commerce Bd. 35, Lohmer

Matthäus, W.-G. (2007): Statistische Tests mit Excel leicht erklärt: Beurteilende Statistik für jedermann, Braunschweig

Maurer, M./Jandura, O. (2009): Masse statt Klasse? Einige kritische Anmerkungen zu Repräsentativität und Validität von Online-Befragungen, in: Jackob, N./Schoen, H./Zerback, T. (Hrsg.): Sozialforschung im Internet: Methodologie und Praxis der Online-Befragung, Wiesbaden
Michon, P. (2007). Quality, preferences, reason, satisfaction: Part Time employment in seven European Union Countries, Amsterdam; online: http://www.wageindicator.org/documents/publicationslist/ WOLIWEBparttime07

(N)ONLINER Atlas (2009): Eine Topographie des digitalen Grabens durch Deutschland: Nutzung und Nichtnutzung des Internets, Strukturen und regionale Verteilung, TNS Infratest, Juni, München Öz, F. (2008): Decent Work and Wageindicator, Oktober, Düsseldorf; online: http://www.wageindicator.org/documents/publicationslist/ publications 2008/081006-decentwork-report.pdf Öz, F./Bispinck, R. (2008a): Was verdienen Bürokaufleute? Eine Analyse von Einkommensdaten auf Basis der WSI-LohnSpiegel-Datenbank, Arbeitspapier 02, Oktober, Düsseldorf

Öz, F./Bispinck, R. (2008b): Was verdienen Ingenieure und Ingenieurinnen? Eine Analyse von Einkommensdaten auf Basis der WSI-LohnSpiegelDatenbank, Arbeitspapier 01, April, Düsseldorf

Öz, F./R. Bispinck, R. (2009a): Was verdienen Bankkaufleute? Eine Analyse von Einkommensdaten auf Basis der WSI-LohnSpiegel-Datenbank, Arbeitspapier 01, Februar, Düsseldorf

Öz, F./Bispinck, R. (2009b): Was verdienen Technikerinnen und Techniker? Eine Analyse von Einkommensdaten auf Basis der WSI-LohnSpiegelDatenbank, Arbeitspapier 02, Februar, Düsseldorf

Sue, V. M./Ritter, L. A. (2007): Conducting Online Surveys, Los Angeles Tijdens, K. (2004): The Dataset, Measurement Issues and the Methodology of the Dutch Wagelndicator Internet Survey, Amsterdam; online: http://www.wageindicator.org/documents/publicationslist/ wp25datameasurment 